\title{
Efectos de exposición aguda a cadmio en la acción de estrógenos en útero de rata impúber
}

\author{
ANDREI N. TCHERNITCHIN ${ }^{1}$, FRANCISCA OLIVARES $1,3,{ }^{1,}$, CARLOS ARANDA ${ }^{1}$, \\ RODRIGO A. BUSTAMANTE ${ }^{1}$, LEONARDO GAETE ${ }^{1,4, *}$, KARLA FERRADA ${ }^{1,5, *}$, \\ REBECA VILLAGRA ${ }^{1,5, *}$, JEANETTE VERA ${ }^{1,6, *}$, RICARDO J. ITURBE ${ }^{1,7, *}$, YOON A. KIM ${ }^{1,7, *}$, \\ NATALIA B. HERNÁNDEZ ${ }^{1,7, *}$, TINA BIZJAK ${ }^{2, *}$, SPELA NOVSAK ${ }^{2, *}$ \\ 1. ICBM, Facultad de Medicina, Universidad de Chile, Santiago, Chile. \\ 2. Estudiante de Intercambio, Universidad de Graz, Austria. \\ 3. Estudiante Tesista, Tesis de Titulación de Ingeniero Ambiental. \\ 4. Licenciado en Biología, Magíster en Bioquímica y Biología Molecular. \\ 5. Tecnólogo Médico. \\ 6. Tesista de Magister en Salud Pública en curso. \\ 7. Estudiante de Pregrado (Medicina, Universidad de Chile).
}

\begin{abstract}
Effects of acute exposure to cadmiun on response to estrogen in the prepuberal rat uterus

Background: Few information is available about uterine effects of Cadmium $(\mathrm{Cd})$ exposure, where toxic agents affecting the female genital tract interact with estrogen (E) receptors, modifiying myometrial activity and the menstrual cycle, causing dysmenorrhea, infertility and spontaneous abortion. No information exists whether prenatal or early postnatal exposure may cause any gynecologic persistent adverse effect. Our finding of a second mechanism of $\mathrm{E}$ interaction and differences between $\mathrm{E}$ receptors in the various uterine cell types suggests that $\mathrm{Cd}$ may affect differently $\mathrm{E}$ interaction in each cell-type. Objective: Evaluate a possible selective effect of acute $\mathrm{Cd}$ exposure on $\mathrm{E}$ action in the uterus during prepuber age. Method: Female prepuber rats exposed to $\mathrm{Cd} 4 \mathrm{mg} / \mathrm{kg}$ and 2 hours later, treated with Estradiol $-17^{2} 0,3 \mathrm{mg} / \mathrm{kg}$. A myometrial sample was obtained under anesthesia 24 hours after $\mathrm{E}$ treatment and histologically processed for the quantification of $\mathrm{E}$ responses on different uterine cell-types. Results: Cd exposure potentiates Einduced uterine eosinophilia and endometrial edema and inhibits E-induced cell hypertrophy in circular myometrium and cell proliferation in luminal myometrium. $\mathrm{Cd}$, in the absence of hormone stimulation, causes a slight cell hypertrophy in circular myometrium. Conclusions: Acute exposure to $\mathrm{Cd}$ affects differently various responses to $\mathrm{E}$ in the different uterine cell-types. Future studies should verify whether this effect explains Cd-induced infertility, postpubertal sex organ development and whether prenatal or early postnatal exposure to $\mathrm{Cd}$ induces delayed persistent effects.

(Key words: Cadmium, toxicity, uterus, estrogen, estrogen responses, immature rat).

Rev Chil Pediatr 2008; 79 (4): 373-380
\end{abstract}

Trabajo recibido el 12 de noviembre de 2007, devuelto para corregir el 03 de enero de 2008, segunda versión 17 de marzo de 2008, aceptado para publicación 23 de abril de 2008.

Financiamiento: Proyecto de la Sociedad Chilena de Pediatría y Proyecto Multidisciplinario en Temas de Interés Nacional MULT 06/27-2 de la Vicerrectoría de Investigación de la Universidad de Chile.

Correspondencia a:

Andrei N. Tchernitchin V.

E-mail: atcherni@gmail.com, atcherni@med.uchile.cl 


\section{RESUMEN}

Antecedentes: Existe poca información sobre efectos del cadmio $(\mathrm{Cd})$ en el útero. En mujeres altera la actividad miometrial, el ciclo menstrual y causa dismenorrea, abortos espontáneos, infertilidad y mortinatos. No existe información si la exposición prenatal o postnatal temprana causa efectos ginecológicos diferidos persistentes. Los tóxicos que afectan el útero suelen interactuar con receptores de estrógeno (E). Nuestro hallazgo de un segundo mecanismo de acción de E y de diferencias entre receptores de E de los diversos tipos celulares uterinos hacen posible que el $\mathrm{Cd}$ interactúe con los $\mathrm{E}$ en forma diferente en cada tipo celular. Objetivos: Buscar un posible efecto selectivo de la exposición aguda a $\mathrm{Cd}$ con algunas respuestas a E en útero de rata durante la edad prepuberal. Métodos: Ratas hembra impúberes recibieron $4 \mathrm{mg} \mathrm{Cd} / \mathrm{kg} \mathrm{p.c.} \mathrm{y} 2$ $\mathrm{h}$ después se trataron con $0,3 \mathrm{mg}$ estradiol-17 $/ \mathrm{kg}$ p.c.; los úteros fueron obtenidos bajo anestesia a las 24 $\mathrm{h}$ del tratamiento con E. Los úteros se procesaron para la cuantificación de respuestas a $\mathrm{E}$ en cada tipo celular por separado. Resultados: La exposición a Cd incrementa la eosinofilia uterina y edema endometrial inducidos por E; inhibe las siguientes respuestas a E: hipertrofia celular en miometrio circular, proliferación celular en epitelio luminal y miometrio. En ausencia de hormona, el cadmio causa una leve hipertrofia celular en miometrio circular. Conclusiones: La exposición aguda a Cd afecta de manera diferente las respuestas a $\mathrm{E}$ en los diversos tipos celulares uterinos de rata prepuberal. Futuros estudios deberán verificar si este efecto explica la infertilidad causada por exposición a $\mathrm{Cd}$, afecta el desarrollo postpuberal de los órganos sexuales, e investigar si la exposición prenatal o postnatal temprana induce efectos diferidos persistentes, como puede ocurrir en población infantil prenatalmente expuesta a $\mathrm{Cd}$.

(Palabras clave: toxicidad, cadmio, útero, estrógeno, respuestas estrogénicas, rata impúber).

Rev Chil Pediatr 2008; 79 (4): 373-380

\section{Introducción}

El cadmio $(\mathrm{Cd})$ es un metal pesado que presenta toxicidad sobre el ser humano. Sus principales orígenes son antropogénicos. Niveles altos de $\mathrm{Cd}$ se asocian a graves efectos sobre la salud, en tanto la exposición a niveles menores se asocia a cáncer pulmonar y prostático ${ }^{1,2}$, insuficiencia renal y enfermedades cardiovasculares ${ }^{3}$, alteraciones del metabolismo de la vitamina $\mathrm{C}$ y trastornos gastrointestinales agudos. Varios estudios atribuyen al $\mathrm{Cd}$ alteraciones genéticas, entre ellas aberraciones cromosómicas e hipoploidía.

En hamsters hembra, el Cd inhibe la ovulación y produce esterilidad ${ }^{4}$; en ratas causa cambios patológicos en útero ${ }^{5}$, ovarios ${ }^{6}$ y placenta $^{7}$. En la mujer, afecta la actividad miometrial en ausencia de embarazo ${ }^{8}$, altera el ciclo menstrual, causa dismenorrea, esterilidad, abortos espontáneos, mortinatos ${ }^{9}$, daño placentario ${ }^{10} \mathrm{y}$ aumenta el nivel de testosterona en suero ${ }^{11}$.

$\mathrm{Se}$ ha sugerido que el $\mathrm{Cd}$ activa receptores citosólico-nucleares de estrógeno $(\mathrm{E})^{12}$ (ver comentario editorial de S. Safe ${ }^{13}$ ), y que en ausencia de hormona este metal pesado repro- duce los efectos de $\mathrm{E}$ tanto en útero como en glándula mamaria ${ }^{14}$. En células tumorales mamarias MCF7 el Cd activaría receptores de $\mathrm{E}$ alfa $\mathrm{y}$ beta $^{15}$. Se ha sugerido que el $\mathrm{Cd}$ interactúa con grupos tiol ( $\mathrm{SH})$ del sitio activo o de unión del receptor de $\mathrm{E}$ a la hormona ${ }^{16}$. Este hecho es importante porque no todos los receptores de $\mathrm{E}$ presentan grupos $\mathrm{SH}$ en su sitio activo; a diferencia de los citosólico-nucleares que son afectados por los bloqueadores de grupos $\mathrm{SH}^{17}$, los receptores de $\mathrm{E}$ de los eosinófilos no lo son ${ }^{18,19}$. En consecuencia, es posible esperar una disociación entre las diversas respuestas a E bajo el efecto de exposición aguda a $\mathrm{Cd}$.

Si todos los receptores de $\mathrm{E}$ fueran idénticos entre sí y existiera un único mecanismo de acción hormonal, tal como se consideró inicialmente ${ }^{17}$, sería suficiente investigar sólo una respuesta estrogénica para verificar si el $\mathrm{Cd}$ afecta o no la acción de los E.

El hallazgo ${ }^{20,21}$ y posteriores estudios ${ }^{19,22}$, en nuestro laboratorio, de receptores de $\mathrm{E}$ en la superficie de los leucocitos eosinófilos, nos ha permitido la descripción ${ }^{22-25}$ del primero de los mecanismos no genómicos de acción de hor- 
monas esteroidales, involucrado en diversas respuestas estrogénicas denominadas no-genómicas. Los receptores clásicos "citosólico-nucleares" descritos por Jensen ${ }^{17}$, por el contrario, están involucrados en las "respuestas genómicas" a los E (hipertrofia de los diversos tipos celulares uterinos, síntesis de proteínas específicas, respuesta proliferativa para diversos tipos celulares, etc) mediante el mecanismo de activación (desrepresión) genómica. Hoy día también se conocen varias diferencias entre los receptores citosólico-nucleares de $\mathrm{E}$ de los diversos tipos celulares de órganos blanco ${ }^{19,26-28}$. Estos antecedentes sugieren la posibilidad de una disociación toxicológica de respuestas a $\mathrm{E}$, lo cual justifica la necesidad de investigar posibles efectos selectivos de la exposición a $\mathrm{Cd}$ en algunas respuestas hormonales en órganos blanco.

El presente trabajo representa la primera etapa del estudio y describe efectos precoces de exposición aguda a cadmio; en etapas posteriores se investigarán los efectos diferidos de la exposición perinatal a dicho tóxico por la posibilidad que causen efectos diferidos persistentes por el mecanismo del imprinting ${ }^{29}$.

\section{Material y Métodos}

Ratas hembra Sprague Dawley prepúberes (21 días de edad) fueron expuestas a $\mathrm{Cd}$ (una dosis de $4 \mathrm{mg} / \mathrm{kg}$ p.c., vía subcutánea en el dorso del animal) o a vehículo. La dosis elegida se basó en los estudios de Baranski y Sitarek ${ }^{30}$. Dos horas después de la exposición, las ratas se trataron con estradiol-17 $\beta(300 \mu \mathrm{g} / \mathrm{kg}$ peso corporal) o vehículo, y a las 24 horas del tratamiento, se extrajeron los úteros bajo anestesia, se los fijó en formalina neutra al $4 \%$ para posterior proceso histológico ${ }^{23,31}$. Luego los animales fueron eutanasiados por sobredosis de anestésico. Se utilizaron 10 animales por condición experimental, total 40 animales.

Se evaluaron respuestas estrogénicas no genómicas (eosinofilia y edema uterinos) y genómicas (hipertrofia miometrial y proliferación celular en epitelio luminal uterino y miometrio). La eosinofilia uterina fue evaluada por recuento en 30 cortes transversales de útero por animal ${ }^{23,31}$; el edema del endometrio profundo fue evaluado por morfometría en 36 áreas en 12 cortes transversales de útero ${ }^{31}$; la hipertrofia miometrial fue evaluada por morfometría en 36 áreas en 12 cortes transversales de úte$\mathrm{ro}^{31,32}$, y la respuesta proliferativa fue evaluada por recuento de figuras mitóticas en 24 cortes de útero en cada capa histológica uterina por separado y expresada como número de mitosis por corte de útero ${ }^{32}$. Los resultados fueron expresados como \% de la respuesta obtenida con $\mathrm{E}$ en ratas controles, valor que fue definido como $100 \%$ de respuesta, y definiendo el valor obtenido en los controles como $0 \%$ de respuesta, pudiendo existir valores negativos cuando las mediciones reflejaron valores inferiores a los del control ${ }^{33}$.

Análisis Estadístico: Para realizar comparaciones múltiples entre las condiciones experimentales, los datos fueron sometidos al test de la mínima diferencia significativa (LSD). La varianza común para este test fue obtenida por análisis de varianza de entrada simple (ANOVA), y no se declaran diferencias significativas a menos que el test de ANOVA fuera significativo $(\mathrm{p}<0,05)^{34}$.

\section{Resultados}

La figura 1 muestra que la eosinofilia uterina (A) y el edema endometrial (B) inducidos por $\mathrm{E}$ en animales expuestos a $\mathrm{Cd}$ son de mayor intensidad que las respuestas a $\mathrm{E}$ en animales no expuestos a $\mathrm{Cd}(\mathrm{p}<0,0001$ y $\mathrm{p}<0,001$ para ambas respuestas). La figura 2 muestra que la exposición a $\mathrm{Cd}$ inhibe parcialmente la hipertrofia celular inducida por $\mathrm{E}$ en miometrio circular ( $p<0,05$, en comparación con la respuesta a $\mathrm{E}$ animales no expuestos a $\mathrm{Cd}$ ), y en ausencia de hormona, la exposición a $\mathrm{Cd}$ causa una leve hipertrofia celular en este tejido ( $p<0,01$, en comparación con controles no tratados con hormona y no expuestos a $\mathrm{Cd}$ ). La figura 3 muestra que el $\mathrm{Cd}$ inhibe en forma pronunciada la respuesta proliferativa a $E$ en epitelio luminal uterino (A) y en miometrio circular (B) $(\mathrm{p}<0,001$ y $\mathrm{p}<0,0001$ respectivamente, en comparación con la respuesta proliferativa a $\mathrm{E}$ en animales no expuestos a $\mathrm{Cd}$ ). 

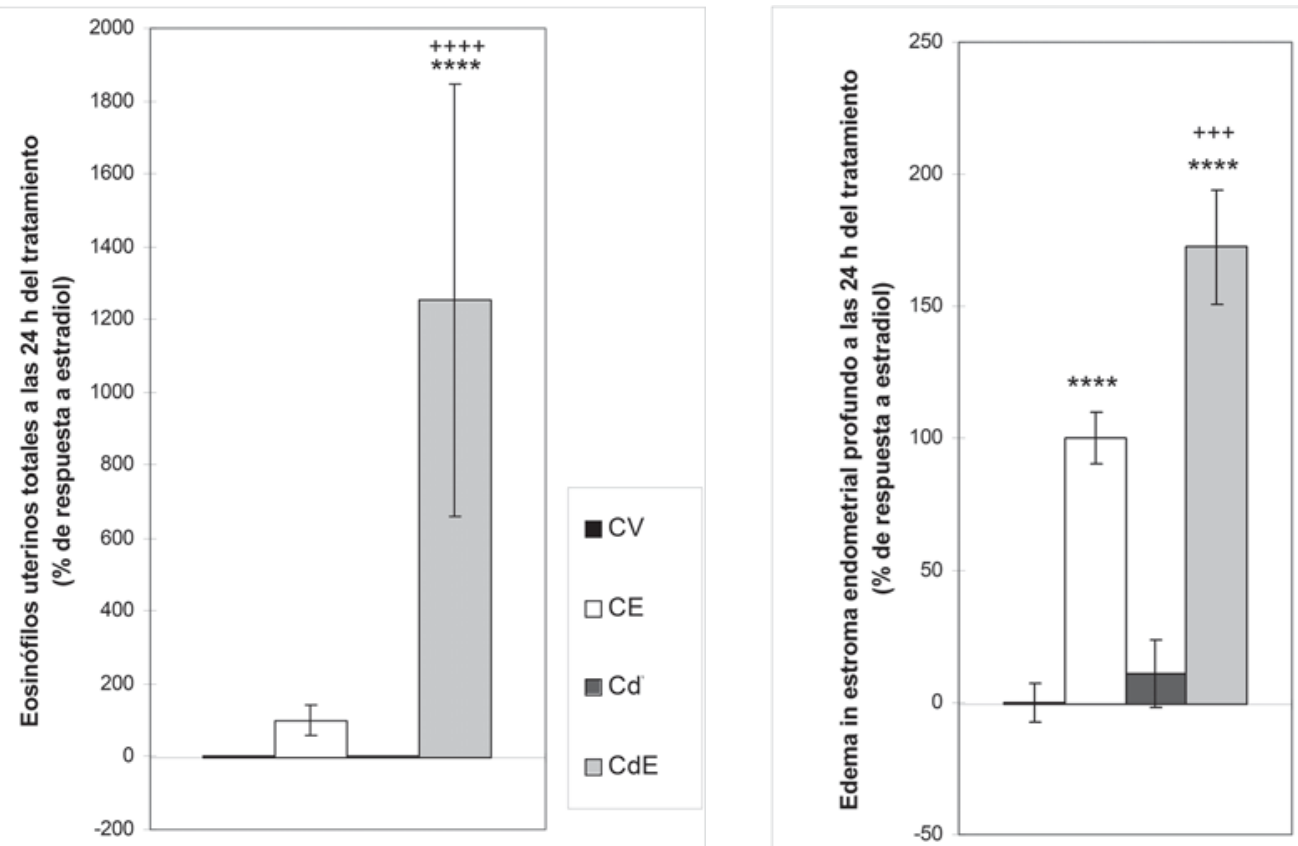

CV24

口CE24

$\square \mathrm{CdV} 24$

$\square \mathrm{CdE} 24$

A

B

Figura 1. Efecto de la exposición aguda a cadmio en la eosinophilia uterina (A) y edema del estroma endometrial profundo (B) inducidos por estradiol $24 \mathrm{~h}$ del tratamiento con la hormona o vehículo. Las ratas fueron expuestas a cadmio s.c. (Cd) o los controles a su vehículo (C), y dos horas después fueron tratadas s.c. con estradiol-17ß (E) o su vehículo (V). Los úteros fueron extraídos bajo anestesia $24 \mathrm{~h}$ después del tratamiento con E o su vehículo. Las barras indican los promedios (expresados como $\%$ de la respuesta a $\mathrm{E}$ en animales no expuestos a Cd) \pm el error estándar del promedio. Estadística: Test de la mínima diferencia significativa (LSD), $* * * * \mathrm{p}<0,0001$, comparado con la condición homóloga sin tratamiento con $\mathrm{E} ;+++\mathrm{p}>0,001$; $++++\mathrm{p}<0,0001$, comparado con la condición homóloga no expuesta a $\mathrm{Cd}$.

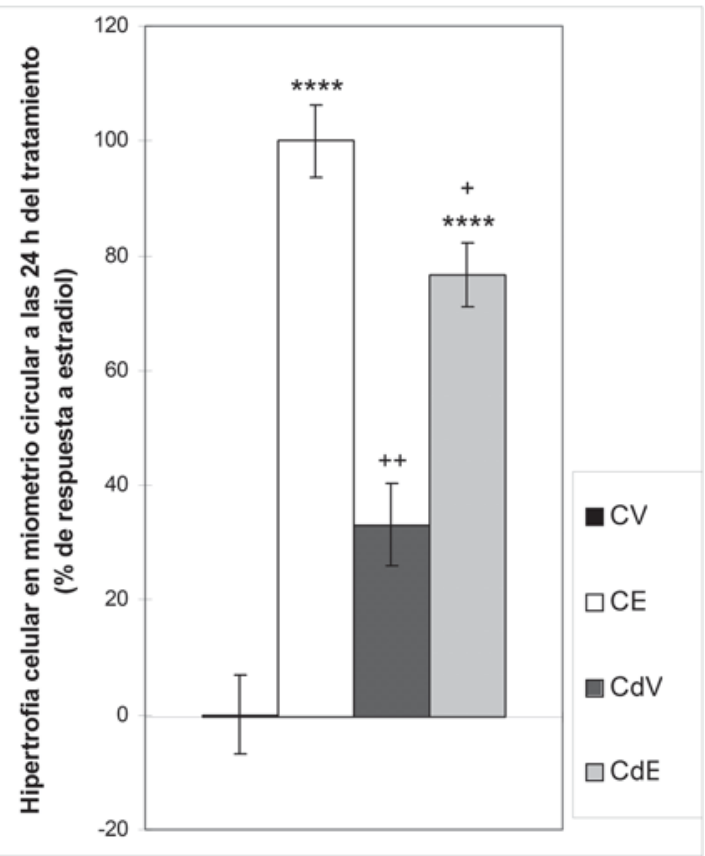

Figura 2. Efecto de la exposición aguda a cadmio en la hipertrofia celular en miometrio circular inducida por estradiol $24 \mathrm{~h}$ del tratamiento con la hormona o vehículo. Las ratas fueron expuestas a cadmio s.c. (Cd) o los controles a su vehículo $(\mathrm{C})$, y dos horas después fueron tratadas s.c. con estradiol-17 $\beta$ (E) o su vehículo (V). Los úteros fueron extraídos bajo anestesia $24 \mathrm{~h}$ después del tratamiento. Las barras indican los promedios (expresados como \% de la respuesta a $\mathrm{E}$ en animales no expuestos a Cd) \pm el error estándar del promedio. Estadística: Test de la mínima diferencia significativa (LSD), $* * * * \mathrm{p}<0,0001$, comparado con la condición homóloga sin tratamiento con $\mathrm{E} ;+\mathrm{p}>0,05,++\mathrm{p}<0,01$, comparado con la condición homóloga no expuesta a $\mathrm{Cd}$. 

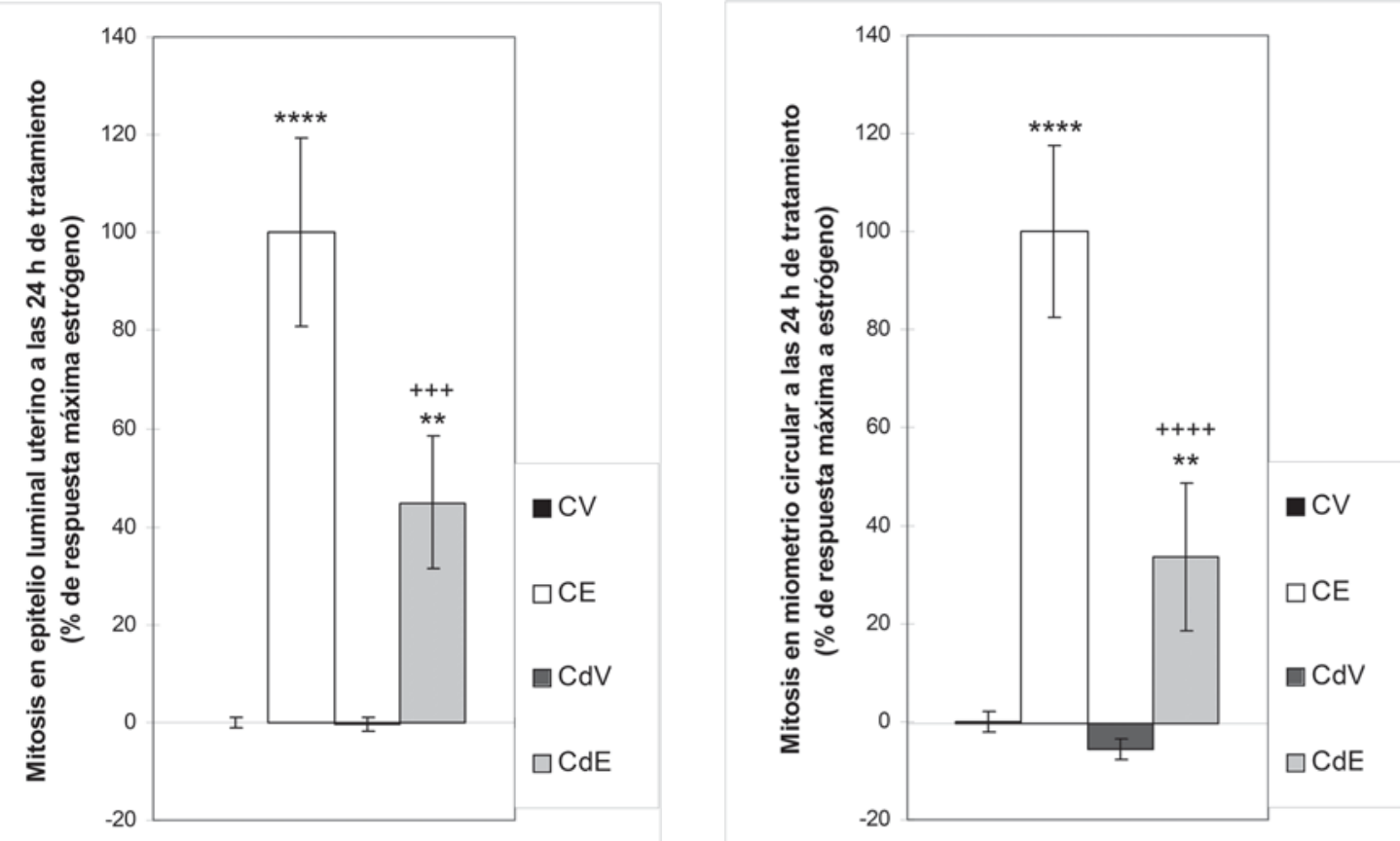

A

B

Figura 3. Efecto de la exposición aguda a cadmio en la respuesta proliferativa en epitelio luminal uterino (A) y miometrio (B), inducidos por estradiol $24 \mathrm{~h}$ del tratamiento con la hormona o vehículo. Las ratas fueron expuestas a cadmio s.c. (Cd) o los controles a su vehículo (C), y dos horas después fueron tratadas s.c. con estradiol-17 $\beta$ (E) o su vehículo (V). Los úteros fueron extraídos bajo anestesia $24 \mathrm{~h}$ después del tratamiento. Las barras indican los promedios (expresados como \% de la respuesta a E en animales no expuestos a Cd) \pm el error estándar del promedio. Estadística: Test de la mínima diferencia significativa (LSD), $* * \mathrm{p}<0,01, * * * * \mathrm{p}<0,0001$, comparado con la condición homóloga sin tratamiento con $\mathrm{E} ;+++\mathrm{p}>0,001,++++\mathrm{p}<0,0001$, comparado con la condición homóloga no expuesta a $\mathrm{Cd}$.

La figura 4 muestra microfotografias representativas de endometrio profundo y miometrio circular (A, B y C) y epitelio endometrial luminal (D, E y F), en animales controles sin exposición a Cd ni tratamiento con E (A y D), sin exposición a cadmio y tratados con $\mathrm{E}(\mathrm{B} \mathrm{y} \mathrm{E}) \mathrm{y}$ en expuestos a $\mathrm{Cd}$ y tratados con $\mathrm{E}$ ( $\mathrm{C}$ y F). No se muestra la condición expuestos a $\mathrm{Cd}$ y sin tratamiento hormonal, por su similitud a la de animales controles. La eosinofilia uterina y el edema endometrial profundo son de mayor magnitud en animales expuestos a $\mathrm{Cd}$ y tratados con $\mathrm{E}(\mathrm{C})$, que en los no expuestos tratados con E (B); no se observan eosinófilos uterinos ni edema endometrial profundo en animales controles (A). Se observa un mayor número de figuras mitóticas en animales no expuestos tratados con $\mathrm{E}(\mathrm{E})$ que en aquellos expuestos a $\mathrm{Cd}$ $\mathrm{y}$ tratados con $\mathrm{E}(\mathrm{F})$.

\section{Discusión}

Hasta nuestro conocimiento, el presente estudio entrega la primera evidencia experimental que la exposición aguda a $\mathrm{Cd}$ potencia algunas respuestas a $\mathrm{E}$ mientras que inhibe otras. $\mathrm{El}$ pretratamiento con $\mathrm{Cd} 2 \mathrm{~h}$ antes del tratamiento hormonal potencia la eosinofilia y el edema uterinos (respuestas no-genómicas), pero inhibe la proliferación celular en epitelio luminal uterino y miometrio, y la hipertrofia celular en miometrio circular. Esta disociación de respuestas a la hormona, sugiere diferencias en la interacción con cada uno de los dos mecanismos descritos en el útero para la acción de $\mathrm{E}^{19,22,25}$. Considerando el papel de los eosinófilos uterinos en el desarrollo del edema endometrial inducido por $\mathrm{E}^{22,24}$, la potenciación de esta última respuesta por $\mathrm{Cd}$ puede ser explicada por 

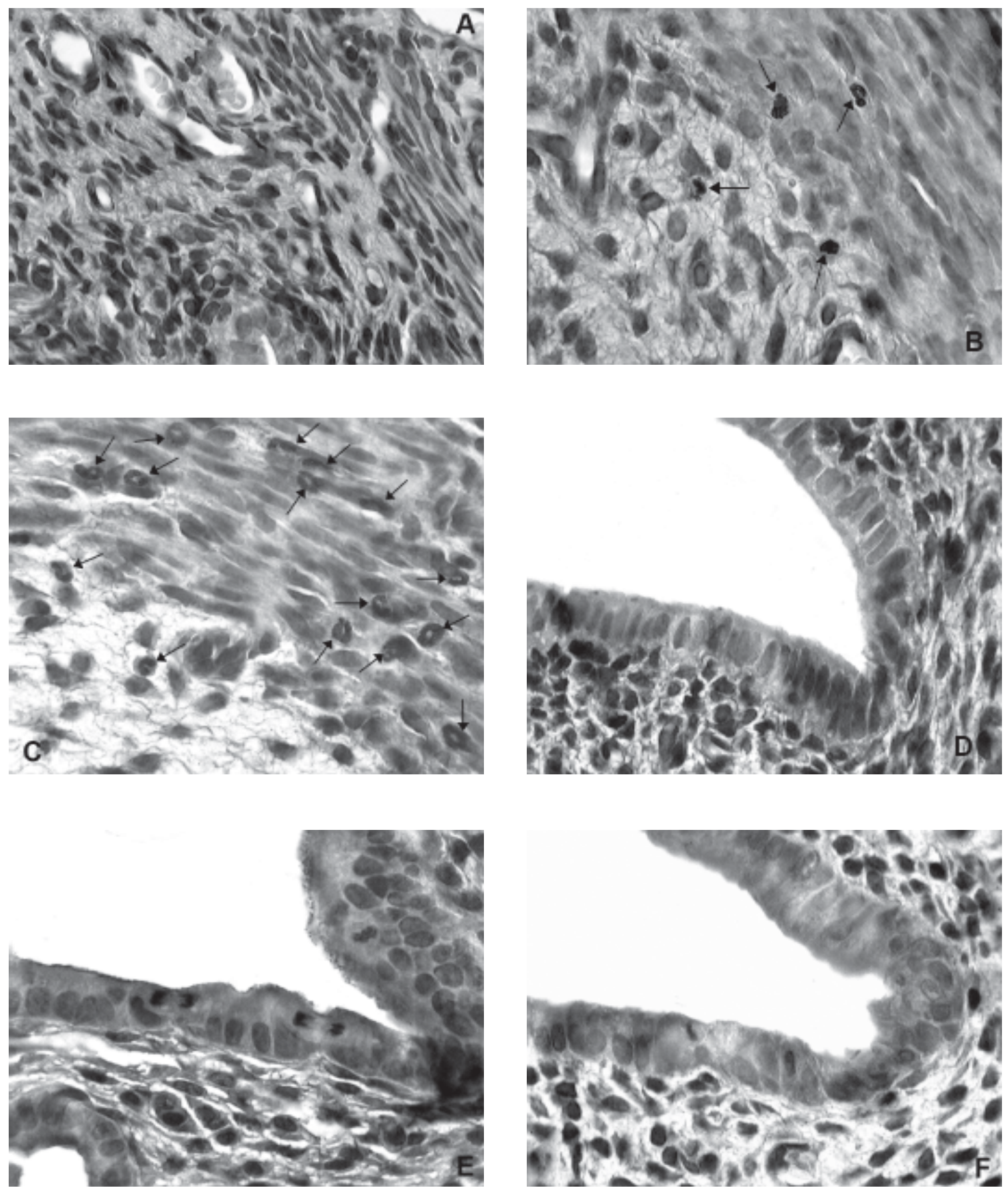

Figura 4. Microfotografías de útero para observar la eosinofilia uterina y edema endometrial inducidos por estrógeno (A, B y C) y de mitosis en epitelio luminal uterino (D, E y F) en animales no expuestos y tratados con E (B y E), y en animales expuestos a $\mathrm{Cd}$ y luego tratados con $\mathrm{E}(\mathrm{C}$ y F), en comparación con animales controles no expuestos y sin tratamiento hormonal (A y D), que no presentan dichas respuestas. Las microfotografías fueron tomadas con objetivo de inmersión 100x. Los eosinófilos uterinos (flechas) se reconocen por el aspecto de sus núcleos muy obscuro y en la mayoría de ellos, núcleo en anillo. El edema se observa como la mayor separación entre los núcleos celulares del estroma endometrial profundo (porción inferior izquierda de las microfotografías). El miometrio circular se observa en la porción derecha superior de las fotografías A, B y C. Las mitosis se observan en el epitelio luminal del endometrio, que recubre el lumen uterino. 
un aumento en el número de eosinófilos uterinos. En concordancia con los presentes resultados, se ha demostrado previamente que toda condición experimental que incremente la eosinofilia uterina $^{22}$, aún en ausencia de $\mathrm{E}^{22}$, incrementa en forma paralela el edema endometrial. Mas aún, se ha reportado una correlación entre eosinofilia e imbibición acuosa uterinos inducidos por $\mathrm{E}^{35}$.

Nuestro estudio no permite discernir si la leve hipertrofia celular en miometrio circular en ratas expuestas a $\mathrm{Cd}$ sin tratamiento con $\mathrm{E}$ es inducida por activación de receptores de $\mathrm{E}$, o por otro mecanismo.

Las disimilitudes entre los efectos de la exposición aguda a cadmio, con aquellos de una exposición a plomo ${ }^{33}$, sugieren diferencias en los mecanismos involucrados. El plomo inhibe la eosinofilia uterina inducida por $\mathrm{E}^{33}$; en cambio el $\mathrm{Cd}$ potencia dicha respuesta hormonal. El plomo antagoniza totalmente la hipertrofia celular inducida por E en miometrio circular ${ }^{33}$, en cambio el Cd sólo la inhibe levemente. Esto puede sugerir que algunos de los mecanismos involucrados en las toxicidad de ambos metales en útero no sean los mismos.

La importante inhibición por $\mathrm{Cd}$ de la respuesta proliferativa a $\mathrm{E}$ en miometrio y epitelio luminal uterino puede contribuir a explicar la infertilidad causada por $\mathrm{Cd}$ tanto en animales de laboratorio ${ }^{4}$ como en la mujer ${ }^{9}$. Una interacción con la acción de $\mathrm{E}$ después de la menarquia puede interferir con el desarrollo de los órganos sexuales y causar secuelas. Esta posibilidad deberá investigarse en estudios epidemiológicos en pacientes pediátricos y en adultos. La diferencia entre la magnitud de la inhibición de la respuesta proliferativa en miometrio y la leve inhibición de la hipertrofia celular en este tejido por $\mathrm{Cd}$, sugieren, pero no demuestran, diferencias entre los receptores involucrados en ambas respuestas o en los mecanismos moleculares involucrados (cofactores, co-inductores alostéricos, fosforilación del receptor, etc). Estas posibles diferencias también deberán ser investigadas.

Numerosos agentes químicos que interactúan en forma aguda con la acción de los $\mathrm{E}^{32,36,37}$, también causan efectos diferidos irreversibles después de una exposición perinatal ${ }^{29,38}$. El mecanismo involucrado es el imprinting o programación celular, que ocurre en períodos críticos del desarrollo fetal tardío, perinatal o infantil temprano, y es responsable del desarrollo de diversas patologías que se manifiestan más tarde en la vida ${ }^{29,38}$, incluidas patologías ginecológicas y alteraciones de la sexualidad ${ }^{39}$. Trabajos en curso en nuestro Laboratorio están destinados a explorar esta posibilidad, que alerta ante la necesidad de evitar la exposición a $\mathrm{Cd}$ durante estos períodos tan sensibles del desarrollo de órganos y tejidos, y que puede ser responsable de diversas patologías gineco-obstétricas de la mujer postpuberal, adolescente o adulta, o que afecten su sexualidad.

Se concluye que la exposición aguda a $\mathrm{Cd}$ afecta de manera diferente las respuestas a $\mathrm{E}$ en los diversos tipos celulares uterinos de rata prepuberal, y que futuros estudios deberán verificar si este efecto contribuye a explicar para la especie humana la infertilidad causada por exposición a $\mathrm{Cd}$, e investigar si la exposición prenatal o postnatal temprana induce efectos diferidos persistentes, como puede ocurrir en población infantil prenatalmente expuesta a $\mathrm{Cd}$.

\section{Referencias}

1.- Goyer RA, Liu J, Waalkes MP: Cadmium and cancer of prostate and testis. Biometals 2004; 17: 555-8.

2.- Sahmoun AE, Case LD, Jackson SA, Schwartz GG: Cadmium and prostate cancer: a critical epidemiologic analysis. Cancer Invest 2005; 23: 256-63.

3.- Nishijo M, Morikawa Y, Nakagawa H, et al: Causes of death and renal tubular dysfunction in residents exposed to cadmium in the environment. Occup Environ Med 2006; 63: 545-50.

4.- Saskena SK, Salmonsen R: Effects of cadmium chloride on ovulation and on induction of sterility in the female golden hamster. Biol Reprod 1983; 29: 249-56.

5.- Copius Peereboom-Stegeman JH, Jongstra-Spaapen E, Leene $W$, et al: The effects of long-term exposure to cadmium on the small blood vessels in the rat uter-us: a light microscopic study. Ecotoxicol Environ Saf 1987; 14: 288-97.

6.- Rehm S, Waalkes MP: Cadmium-induced ovarian toxicity in hamsters, mice, and rats. Fundam Appl Toxicol 1988; 10: 635-47.

7.- Levin AA, Plautz JR, di Sant'Agnese PA, Miller RK: Cadmium: pla-cental mechanisms of fetal toxicity. Placenta 1981; Suppl 3: 303-18.

8.- Sipowicz M, Kostrzewska A, Laudanski T, Akerlund M: Effects of cad-mium on myometrial activity of the nonpregnant human. Interactions with calcium and oxytocin. Acta Obstet Gynecol Scand 1995; 74: 93-6. 
9.- Wang $X$, Tian $J$ : Health risks related to residential exposure to cadmium in Zhenhe County, China. Arch Environ Health 2004; 59: 324-30.

10.- Yang K, Julan L, Rubio F, Sharma A, Guan $H$ : Cadmium reduces 11 beta-hydroxysteroid dehydrogenase type 2 activity and expression in human placental trophoblast cells. Am J Physiol Endocrinol Metab 2006; 290: E135-42.

11.- Nagata C, Nagao Y, Shibuya C, Kashiki Y, Shimizu H: Urinary cad-mium and serum levels of estrogens and androgens in postmenopausal Japanese women. Cancer Epidemiol Biomarkers Prev 2005; 14: 705-8.

12.- Stoica A, Katzenellenbogen BS, Martin MB: Activation of estrogen receptor alpha by the heavy metal cadmium. Mol Endocrinol 2000; 14: 545-53.

13.- Safe $S$ : Cadmium's disguise dupes the estrogen receptor. Nature Med 2003; 9: 1000-1.

14.- Johnson MD, Kenney N, Stoica A, et al: Cadmium mimics the in vivo effects of estrogen in the uterus and mammary gland. Nat Med 2003; 9: 1081-84.

15.- Martínez-Campa C, Alonso-González C, Mediavilla MD, et al: Melatonin inhibits both ER alpha activation and breast cancer cell proliferation induced by a metalloestrogen, cadmium. J Pineal Res 2006; 40: 2916.

16.- Young PC, Cleary RE, Ragan WD: Effect of metal ions on the binding of 17 beta-estradiol to human endometrial cytosol. Fertil Steril 1977; 28: 459-63.

17.- Jensen EV, DeSombre ER: Mechanism of action of the female sex hormones. Annu Rev Biochem 1972; 41: 203-30.

18.- Collao C, Tchernitchin A: Effect of sulphydryl group blockage on estrogen binding by the receptors of the uterine eosinophils. IRCS Med Sci 1976; 4: 87.

19.- Tchernitchin AN, Mena MA, Rodríguez A, Maturana M: Radioauto-graphic localization of estrogen receptors in the rat uterus: a tool for the study of classical and nontraditional mechanisms of hormone action. En: Pertschuk LP, Lee SH, eds. Localization of Putative Steroid Receptors, Vol. 1, Experimental Systems, Boca Raton, Florida, U.S.A.: Editorial CRC Press, 1985; 5-37.

20.- Tchernitchin A: Autoradiographic study of $(6,7)-{ }^{3} \mathrm{H}$ oestradiol-17ß incorporation into rat uterus. Steroids 1967; 10: 661-8.

21.- Tchernitchin A, Tchernitchin X, Robel P, Baulieu EE: Liaison de l'oestradiol dans les leucocytes polinucléaires éosinophiles humains. C R Acad Sci Paris 1975; 280 (Serie D): 1477-80.

22.- Tchernitchin AN, Mena MA, Soto J, Unda C: The role of eosinophils in the action of estrogens and other hormones. Med Sci Res 1989; 17: 5-10.

23.- Tchernitchin A, Roorijck J, Tchernitchin X, Vandenhende J, Galand P: Dramatic early increase in uterine eosinophils after oestrogen administration. Nature 1974; 248: 142-3

24.- Tchernitchin AN: Eosinophil-mediated non-genomic parameters of estrogen stimulation: A separate group of responses mediated by an independent mechanism. J Steroid Biochem 1983; 19: 95-100.
25.- Galand P, Tchernitchin N, Tchernitchin AN: Dissociation of uterine eosinophilia and water imbibition from other estrogen-induced responses by nafoxidine pretreatment. Mol Cell Endocrinol 1985; 42: 227-33.

26.- Kuiper GG, Enmark E, Pelto-Huikko M, Nilsson S, Gustafsson J-A: Cloning of a novel receptor expressed in rat prostate and ovary. Proc Natl Acad Sci USA 1996; 93: 5925-30.

27.- Nilsson S, Mäkelä S, Treuter E, et al: Mechanisms of estrogen action. Physiol Rev 2001; 81: 1535-65.

28.- Sakamoto H, Matsuda KI, Hosokawa K, et al: Expression of $\mathrm{G}$ protein-coupled receptor-30, a $\mathrm{G}$ protein-coupled membrane estrogen receptor, in oxytocin neurons of the rat paraventricular and supraoptic nuclei. Endocrinology 2007; 148: 5842 50 .

29.- Tchernitchin AN, Tchernitchin N: Imprinting of paths of heterodifferentiation by prenatal or neonatal exposure to hormones, pharmaceuticals, pollutants and other agents and conditions. Med Sci Res 1992; 20: 391-397.

30.- Baranski B, Sitarek K: Effect of oral and inhalation expusure to cadmium on the oestrus cycle in rats. Toxicol Lett 1987; 36: 267-73.

31.- Grunert G, Fernández S, Tchernitchin AN: Methods for the evaluation of responses to estrogen in individual cell types or regions of the uterus. Hormone Res 1984; 19: 253-62.

32.- Grunert G, Porcia M, Tchernitchin AN: Differential potency of oestradiol-17ß and diethylstilboestrol on separate groups of responses in the rat uterus. J Endocrinol 1986; 110: 103-14.

33.- Tchernitchin NN, Villagra A, Tchernitchin AN: Antiestrogenic activity of lead. Environ Toxicol Water Qual 1998; 13: 43-53.

34.- Snedecor $G W$, Cochran $W G$ : One way classifications. Analysis of variance. En: Snedecor GW, Cochran WG, eds. Statistical Methods. 6a ed., Ames, Iowa, USA: Editorial Iowa State University, 1967; 258-98.

35.- Tchernitchin AN, Galand P: Oestrogen levels in the blood, not in the uterus, determine uterine eosinophilia and oedema. J Endocrinol 1983; 99: 123-30.

36.- Mena MA, Grunert G, Mansilla MS, et al: Inhibition of non-genomic responses to oestrogen in the rat uterus by testosterone propionate. J Reprod Fertil 1985; 74 : $1-7$

37.- Bustos S, Soto J, Tchernitchin AN: Estrogenic activity of p,p'-DDT. Environ Toxicol Water Qual 1996; 11: 265-71.

38.- Tchernitchin AN, Tchernitchin NN, Mena MA, Unda C, Soto J: Imprinting: Perinatal exposures cause the development of diseases during the adult age. Acta Biol Hung 1999; 50: 425-40.

39.- Tchernitchin AN, Mena MA: Efectos diferidos de contaminantes ambientales y otros agentes en salud reproductiva y sexualidad: un desafío pendiente de la toxicología de la reproducción para la salud de las futuras generaciones. Cuad Med Soc (Chile) 2006; 46: 176-94. 